\title{
THEMATIC PAPERS \\ Child sexual abuse: perspectives from around the world
}

\author{
David Skuse
}

Behavioural and Brain Sciences Unit Institute of Child Health, London, UK, email d.skuse@ ucl.ac.uk
Child sexual abuse is a topic rarely out of the headlines in the UK nowadays. What are the longer-term consequences of such abuse in different cultures? Surprisingly little has been published on the subject. In this issue of International Psychiatry we have commissioned three articles from diverse countries where the problem is often ignored for cultural reasons. It is worth noting that until the late 1970s this was true for the UK too. At that time, a colleague of mine from the USA came over to research the subject here, and was told it would be a waste of her time because child sexual abuse did not exist in this country.

Here, we have articles from China, the Arabian Gulf and Southern Africa. Dr Ko Ling Chan and colleagues from Hong Kong describe their welldesigned survey of over 18000 adolescents from multiple regions of China. A surprising finding is that proportionately more boys than girls reported both contact and non-contact abuse. There were important mental and physical health consequences, but the level of awareness of abuse in the general population was very low.

In the Arabian Gulf, matters of sexual conduct are rarely discussed in public. Drs Ohaeri and
Al-Fayez discuss the findings from a survey of over 4000 Kuwaiti adolescents. They found the prevalence of contact abuse was substantially higher than is reported in China, but, as in China, boys more often reported it than girls. There were mental health consequences for victims, but also a pervasive fear of reporting the experience for fear of the personal social reprisals.

Finally, Neil Andersson discusses the results of a series of studies they conducted in many African countries, using sophisticated survey techniques. The sample of nearly 50000 children, in early adolescence, reported high levels of coerced sex, with little or no difference in prevalence between male and female abuse. It is worrying to discover that abused children were likely to admit to forcing other children into sexual activity, and that disdain for the safety of others through the deliberate spreading of HIV infection was often reported too.

There do appear to be cultural differences in the exposure of children to sexual abuse but, in all the areas surveyed, the problem affects a significant minority of both boys and girls. The longer-term mental health consequences are substantial.

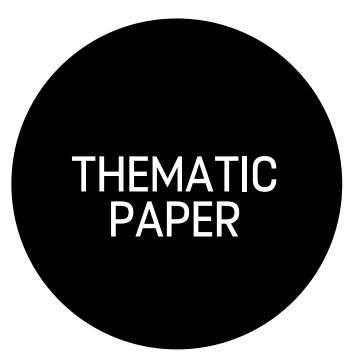

\section{CHILD SEXUAL ABUSE}

\section{Child sexual abuse and health outcomes in the Chinese context}

\author{
Ko Ling Chan ${ }^{1}$ PhD, Elsie Yan ${ }^{1}$ PhD, Daniel Y. T. Fong ${ }^{2}$ PhD, Agnes Tiwari ${ }^{2}$ PhD and \\ Wing Cheong Leung ${ }^{3} \mathrm{PhD}$
}

'Department of Social Work and Social Administration, Hong Kong, Hong Kong, email eklchan@ hku.hk

${ }^{2}$ School of Nursing, Li Ka Shing Faculty of Medicine, The University of Hong Kong. Hong Kong

${ }^{3}$ Department of Obstetrics and Gynaecology, Kwong Wah Hospital, Hong Kong

The Optimus Study was initiated and funded by the UBS Optimus Foundation.
Reported rates of child sexual abuse in China fall at the lower end of the range found in Western studies. However, most of the studies were conducted in only one city or province and thus their results may not be generalisable. Acknowledging the infeasibility of recruiting a truly representative sample, we conducted a survey during 2009-10 using a probability sampling procedure to obtain a large and diverse sample of school-aged adolescents from six regions in China. About one in every 13 children had had experience of sexual abuse. Routine screening in medical and social settings is urged. Efforts should be made to ensure wide awareness of this issue.
As researchers around the world pay increasing attention to child sexual abuse (CSA), the number of studies on CSA in Chinese populations has increased. Reported prevalence rates of CSA in China range from $2 \%$ to $14 \%$ (e.g. Chen et al, 2006; Leung et al, 2008), which fall at the lower end of the range found in Western studies. However, most of these Chinese studies were conducted in only one city or province, and thus their results may not be generalisable. Indeed, this problem was noted by Chen et al (2006), who reported that Chinese studies on CSA were often preliminary and focused on only a specific group (e.g. female adolescents). Yet, given China's huge population and geographical size, it would be extremely challenging to recruit a truly representative national sample. 